\title{
Co-morbid anaemia and stunting among children of pre-school age in low- and middle-income countries: a syndemic
}

\author{
Thach Duc Tran ${ }^{1, *}$, Beverley-Ann Biggs ${ }^{2}$, Sara Holton ${ }^{1}$, Hau Thi Minh Nguyen ${ }^{1}$, \\ Sarah Hanieh ${ }^{2}$ and Jane Fisher ${ }^{1}$ \\ 'Jean Hailes Research Unit, School of Public Health and Preventive Medicine, Monash University, 553 St Kilda Road, \\ Melbourne, VIC 3004, Australia: ${ }^{2}$ Department of Medicine (Royal Melbourne Hospital) at the Doherty Institute, The \\ University of Melbourne, Melbourne, Victoria, Australia
}

Submitted 16 January 2018: Final revision received 27 July 2018: Accepted 7 August 2018: First published online 24 September 2018

\begin{abstract}
Objective: To determine the prevalence of co-morbidity of two important global health challenges, anaemia and stunting, among children aged 6-59 months in low- and middle-income countries.

Design: Secondary analysis of data from Demographic and Health Surveys (DHS) conducted 2005-2015. Child stunting and anaemia were defined using current WHO classifications. Sociodemographic characteristics of children with anaemia, stunting and co-morbidity of these conditions were compared with those of 'healthy' children in the sample (children who were not stunted and not anaemic) using multiple logistic models.

Setting: Low- and middle-income countries.

Subjects: Children aged 6-59 months.

Results: Data from 193065 children from forty-three countries were included. The pooled proportion of co-morbid anaemia and stunting was 21.5 (95\% CI 21.2, $21.9) \%$, ranging from the lowest in Albania (2.6\%; $95 \%$ CI $1.8,3.7 \%$ ) to the highest in Yemen $(43 \cdot 3$; $95 \%$ CI 40.6, 46.1\%). Compared with the healthy group, children with co-morbidity were more likely to be living in rural areas, have mothers or main carers with lower educational levels and to live in poorer households. Inequality in children who had both anaemia and stunting was apparent in all countries.

Conclusions: Co-morbid anaemia and stunting among young children is highly prevalent in low- and middle-income countries, especially among more disadvantaged children. It is suggested that they be considered under a syndemic framework, the Childhood Anaemia and Stunting (CHAS) Syndemic, which acknowledges the interacting nature of these diseases and the social and environmental factors that promote their negative interaction.
\end{abstract}

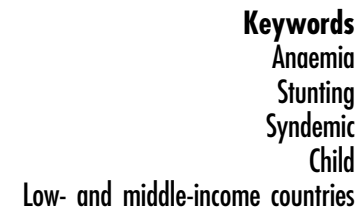

eywords

Stunting Syndemic

Child

Low- and middle-income countries
Anaemia and stunting remain significant early childhood public health challenges in resource-constrained settings ${ }^{(1)}$. Anaemia is defined as having a low $\mathrm{Hb}$ concentration or red blood cell mass, and may be the result of nutritional factors (e.g. deficiencies of iron, folic acid, vitamin $\mathrm{B}_{12}$ and vitamin $\mathrm{A}$ ), infectious diseases (e.g. soiltransmitted helminths, malaria, tuberculosis and HIV/ AIDS) and genetic factors (e.g. thalassaemia) ${ }^{(2)}$. Stunting (height-for-age $Z$-score below WHO growth standards, reflecting slowing of skeletal growth) is widely considered to be a reliable indicator of long-term undernutrition in early childhood ${ }^{(3)}$.

A syndemic, a concept developed by medical anthropologists $^{(4)}$, has been increasingly recognised as a powerful innovative framework to understand, prevent and treat co-occurrence of health burdens. A syndemic has three core features: (i) clustering of two (or more) diseases or health conditions within a specific population; (ii) contextual and social factors that create the conditions in which the two (or more) diseases or health conditions cluster; and (iii) adverse disease interaction resulting from the clustering which increases the health burden among affected populations ${ }^{(5)}$. The syndemic framework refers not only to the cooccurrence of health problems but also acknowledges social and environmental factors such as poverty, low education, social trauma, environmental threats and limited access to health-care which exacerbate the biological interactions among - and the outcomes of - clustered conditions. The 
syndemic framework makes a valuable contribution in assisting researchers, clinical practitioners and policy makers to conceptualise and address health inequities and inequalities across populations.

The co-morbidity of anaemia and stunting in early childhood in resource-constrained settings can be considered using a syndemic approach. There are shared risk factors for these health conditions including immediate causes, such as insufficient access to nutrition and exposure to infectious diseases; underlying causes including household food insecurity, inadequate care, unhealthy household environments and lack of health services; and basic causes including limited resources at community and country levels ${ }^{(1,2)}$. Both anaemia and stunting during early life can lead to significant long-term health, development and social consequences later in life. Data from five birth cohort studies in Brazil, Guatemala, India, the Philippines and South Africa showed that children with low height-forage $Z$-score at 2 years old had shorter adult height, lower educational attainment, reduced adult income, and that their own offspring had decreased birth weight ${ }^{(3,6)}$. Longitudinal studies have shown that $\mathrm{Hb}$ levels in early childhood are linked to cognitive development and school achievement in later childhood $^{(7)}$. A review of twenty-nine studies confirmed that the biological mechanisms of the adverse effect of anaemia on work capacity are consistently strong ${ }^{(8)}$. To date, the prevalence of and risk factors for co-occurrence of these two conditions in early childhood have not been documented.

The aims of the present study were to: (i) determine the prevalence of co-morbid anaemia and stunting among children aged 6-59 months in low- and middle-income countries; (ii) examine the association of these conditions with important sociodemographic characteristics; and (iii) identify socio-economic inequalities among children experiencing these co-morbid conditions.

\section{Methods}

The present study is a secondary analysis using data from Demographic and Health Surveys (DHS) conducted from 2005 to 2015.

\section{Demographic and Health Surveys}

The DHS Program was established in 1984 to advance global understanding of health and population trends in low- and middle-income countries (see more at http://www.dhspro gram.com). To date, more than 300 household surveys have been conducted in over ninety countries. In each survey in a country, a large sample comprising between 5000 and 30000 households was selected using a nationally representative multistage sampling procedure.

Household socio-economic and demographic characteristics were collected by national research teams in home visits using face-to-face structured interviews. The weight, height and $\mathrm{Hb}$ concentration of children aged 6-59 months in all selected households were assessed. Children's weight was measured using a SECA 874 digital scale with $0.01 \mathrm{~kg}$ increments; length (children aged $<2$ years) and height (children aged $\geq 2$ years) were measured using the Shorr height board; and the assessment of $\mathrm{Hb}$ was performed using a HemoCue photometer ( $\mathrm{Hb}$ $201+)^{(9)}$.

\section{Study participants}

The current analysis includes children for whom data about weight, height and $\mathrm{Hb}$ concentration were available for public use and collected between 2005 and 2015. If data from more than one survey were available for one country during that period, only the data from the latest survey were included in the analyses.

\section{Outcomes}

Anaemia was defined as $\mathrm{Hb}$, adjusted for altitude, of less than $110 \mathrm{~g} / \mathrm{l}$ in children aged 6-59 months, according to the WHO classification ${ }^{(10)}$

Child length/height-for-age $Z$-scores were calculated based on child sex, age and length (if aged $<2$ years) or height (if aged $\geq 2$ years) using WHO Anthro software version $3.2 .2^{(11)}$. Stunting was defined as length/heightfor-age $Z$-score $<-2$.

An index of household wealth was constructed by the DHS $^{(9)}$ for each survey independently using the World Bank method ${ }^{(12)}$, which is based on household characteristics including the main materials of the household dwelling's floor, roof and exterior walls; main type(s) of fuel used for cooking; source of drinking-water; type of sanitation facility; and household assets.

Country income classifications, i.e. low income, lowermiddle income, upper-middle income and high income, at the time data were collected were derived from the World Bank's classifications for that year. The groups are based on gross national income per capita. In 2016, low income represents an income of up to \$US 1025; lower-middle income, \$US 1026-4035; upper-middle income, \$US 403612 475; and high income, \$US 12476 or more ${ }^{(13)}$.

\section{Statistical analysis}

All analyses were conducted using survey commands (SVY) in the statistical software package Stata version 14. SVY commands are designed to analyse complex survey data and to adjust standard errors for survey design including cluster sampling and sampling weight ${ }^{(14)}$. In every DHS study, participants were selected using cluster sampling and the cluster codes are provided in the data sets. Cluster sampling weights that are equal (or proportional) to the inverse of the probability of being sampled are calculated for each survey and provided. Pooled 
analyses of data from all countries also included the poststratification weights which adjust for the number of children from each country included in the analysis to ensure the equal contribution of each country. The poststratification weight of a country is the inverse ratio between the number of children included in the analysis of that country and the average number of children included in the analysis per country.

Prevalence of children experiencing co-morbid anaemia and stunting was calculated by country and shown on the world map (source: http://www.qgis.osgeo.org) with different colour codes for ease of visual comparison. The prevalence of anaemia, stunting and co-morbidity for the poorest and richest quintile groups was calculated for each country to examine inequality in child malnutrition among countries. The trends of anaemia, stunting and their comorbidity by child age are presented using the pooled prevalence of the conditions by child age (1-month increments).

The sociodemographic characteristics of children with anaemia only, stunting only and 'healthy' children were compared with those of children with co-morbidity in the sample using multinomial logistic regression analysis. Healthy children were defined as those who were not stunted and did not have anaemia. The comparisons were performed using multiple logistic models with the covariates of child age and sex, mother's (or the main carer's, if the mother was not living with the child) highest education level, household wealth index quintile and place of residence (in an urban or rural area). The wealth index is a continuous indicator and was converted into quintiles by DHS. The lowest quintile is the poorest $20 \%$ and the highest is the richest $20 \%$ of household wealth distribution.

The index of socio-economic inequality in child morbid anaemia and stunting was calculated for each country using the concentration index ${ }^{(12)}$. The concentration index is the summary of the information from the concentration curve showing the relationship between a health variable (in the present study, morbid anaemia and stunting) and the rank of the living standards variable (in the present study, household wealth index). The concentration index is defined as twice the area between the concentration curve and the $45^{\circ}$ line (the line of equality). The concentration index ranges from -1 to 1 , with a zero value indicating no socio-economic-related inequality, a negative value (when the curve lies above the line of equality) indicating disproportionate concentration of the health variable among the poor and a positive value (when it lies below the line of equality) indicating a disproportionate concentration of the health variable among the rich.

\section{Results}

\section{Sample}

Overall, data from 193065 children aged 6-59 months from forty-three low- and middle-income countries were included in the analyses. The characteristics of the included children are summarised by country in the online supplementary material, Supplemental Tables 1-3. The overall proportion of girls in the sample was $49.1 \%$, ranging from $46.4 \%$ in Armenia to $52.9 \%$ in Albania. The mean age of the entire sample was 32.4 months, ranging from 31.4 months in Cote d'Ivoire and Egypt to 35.0 months in Albania. More than two-thirds (69.3\%) of the children in the sample lived in a rural area, with the lowest prevalence in Jordan $(30 \cdot 8 \%)$ and the highest in Malawi (90.2\%).

\section{Prevalence of anaemia, stunting and co-morbidity}

The pooled proportion of anaemia among the children was 56.4 (95\% CI 56.0, 56.9)\%, with the lowest proportion found in Albania (17.3\%; 95\% CI 14.6, 20.3\%) and the highest proportion in Burkina Faso (87.7\%; $95 \%$ CI 86.4, $88.8 \%)$. The pooled proportion of stunting was $34.8(95 \%$ CI $34.4,35.2) \%$, ranging from the lowest in Jordan $(7.6 \%$; $95 \%$ CI $6.5,8.9 \%)$ to the highest in Burundi (61.6\%; $95 \%$ CI $59 \cdot 6,63.7 \%)$. The pooled proportion of co-morbid anaemia and stunting was 21.5 (95\% CI $21 \cdot 2,21.9) \%$, ranging from the lowest in Albania (2.6\%; $95 \%$ CI 1.8 , $3.7 \%)$ to the highest in Yemen $(43.3 \%$; $95 \%$ CI 40.6 , $46 \cdot 1 \%)$. Figure 1 shows the proportion of co-morbidity among children in each country included in the study. Further details on the proportions of anaemia, stunting and co-morbidity by country and country income classifications are presented in Supplemental Table 4 and by demographic characteristics in Supplemental Table 5 (see online supplementary material).

\section{Characteristics of children with anaemia, stunting and co-morbidity}

The results of the multinomial logistic modelling analysis are presented in Table 1 . It shows that boys were less likely to have anaemia alone ( $16 \%$ less likely) or stunting alone ( $7 \%$ less likely) or to be healthy ( $22 \%$ less likely) than to have co-morbid anaemia and stunting. The risk of co-morbidity relative to anaemia alone was lowest in the youngest group (about $60 \%$ less likely) and higher but fairly stable in the older age groups. The risk of comorbidity relative to stunting alone decreased constantly by age group such that the oldest group ( $>4-5$ years) was 3.44 times less likely than the youngest group ( $0.5-1$ year). The risk of co-morbidity relative to being healthy had a U-shaped association with child age. Children from 1 to 2 years of age had the highest risk of co-morbidity relative to being healthy (39\% more likely than children aged $0 \cdot 5-1$ year and more than two times more likely than children aged $4-5$ years). Children living in rural areas were more likely to have co-morbidity than to have anaemia alone ( $34 \%$ more likely) or to be healthy ( $28 \%$ ) but less likely to have co-morbidity than to have stunting alone (16\%). This shows that the risk difference between rural and urban 


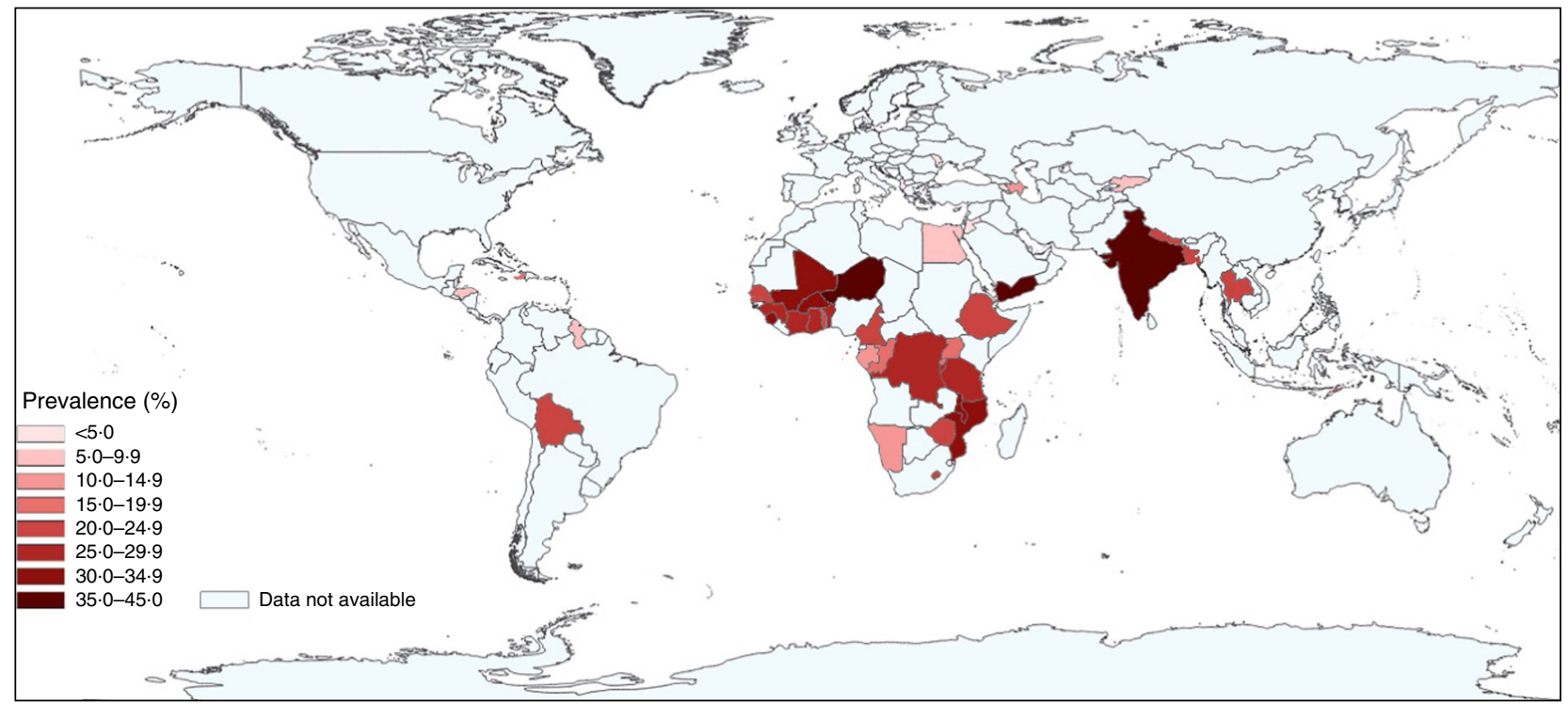

Fig. 1 (colour online) Prevalence of co-morbid anaemia and stunting in children aged 6-59 months ( $n$ 193 065) in forty-three lowand middle-income countries (Demographic and Health Survey data from 2005 to 2015)

Table 1 Multinomial logistic regression model, comparing the sociodemographic characteristics of children with anaemia, stunting and comorbidity of these conditions with those of 'healthy' children (who were not stunted and not anaemic), among children aged 6-59 months ( $n$ 193065) in forty-three low- and middle-income countries (pooled data from forty-three Demographic and Health Survey data sets, 2005 to 2015$)$

\begin{tabular}{|c|c|c|c|c|c|c|}
\hline & \multicolumn{2}{|c|}{$\begin{array}{c}\text { Anaemia only } v \text {. co-morbidity } \\
\text { (ref.) }\end{array}$} & \multicolumn{2}{|c|}{$\begin{array}{l}\text { Stunting only } v \text {. co-morbidity } \\
\text { (ref.) }\end{array}$} & \multicolumn{2}{|c|}{$\begin{array}{l}\text { Healthy v. co-morbidity } \\
\text { (ref.) }\end{array}$} \\
\hline & RRR & $95 \% \mathrm{Cl}$ & RRR & $95 \% \mathrm{Cl}$ & RRR & $95 \% \mathrm{Cl}$ \\
\hline \multicolumn{7}{|l|}{ Child sex } \\
\hline Girl & \multicolumn{2}{|c|}{ Ref. } & \multicolumn{2}{|c|}{ Ref. } & \multicolumn{2}{|c|}{ Ref. } \\
\hline Boy & 0.84 & $0.81,0.87$ & 0.93 & $0.89,0.97$ & 0.78 & $0.75,0.81$ \\
\hline \multicolumn{7}{|l|}{ Child age (years) } \\
\hline 0.5 to 1 & \multicolumn{2}{|c|}{ Ref. } & \multicolumn{2}{|c|}{ Ref. } & \multicolumn{2}{|c|}{ Ref. } \\
\hline$>1$ to 2 & 0.41 & $0.38,0.43$ & 1.35 & $1.20,1.52$ & 0.59 & $0.55,0.64$ \\
\hline$>2$ to 3 & 0.32 & $0.30,0.34$ & 2.05 & $1.83,2.29$ & 0.87 & $0.80,0.94$ \\
\hline$>3$ to 4 & 0.34 & $0.32,0.36$ & 2.69 & $2 \cdot 40,3.01$ & 1.27 & $1.18,1.37$ \\
\hline$>4$ to 5 & 0.41 & $0.38,0.44$ & 3.44 & $3.08,3.86$ & 1.86 & $1 \cdot 72,2 \cdot 01$ \\
\hline \multicolumn{7}{|l|}{ Place of residence } \\
\hline Urban & \multicolumn{2}{|c|}{ Ref. } & \multicolumn{2}{|c|}{ Ref. } & \multicolumn{2}{|c|}{ Ref. } \\
\hline Rural & 0.66 & $0.62,0.70$ & $1 \cdot 16$ & $1.07,1.27$ & 0.72 & $0.67,0.77$ \\
\hline \multicolumn{7}{|l|}{ Mother's/career's highest education level } \\
\hline Secondary or higher & \multicolumn{2}{|c|}{ Ref. } & \multicolumn{2}{|c|}{ Ref. } & \multicolumn{2}{|c|}{ Ref. } \\
\hline Primary & 0.61 & $0.58,0.64$ & 0.85 & $0.79,0.91$ & 0.43 & $0.41,0.46$ \\
\hline No formal education & 0.52 & $0.50,0.55$ & 0.49 & $0.46,0.53$ & 0.20 & $0.19,0.21$ \\
\hline \multicolumn{7}{|l|}{ Household wealth index } \\
\hline Riches quintile & \multicolumn{2}{|c|}{ Ref. } & \multicolumn{2}{|c|}{ Ref. } & \multicolumn{2}{|c|}{ Ref. } \\
\hline Fourth quintile & 0.78 & $0.72,0.85$ & 0.82 & $0.74,0.90$ & 0.71 & $0.65,0.77$ \\
\hline Middle quintile & 0.74 & $0.68,0.80$ & 0.74 & $0.67,0.82$ & 0.62 & $0.57,0.68$ \\
\hline Second quintile & 0.68 & $0.62,0.74$ & 0.66 & $0.60,0.74$ & 0.55 & $0.50,0.60$ \\
\hline Poorest quintile & 0.60 & $0.55,0.65$ & 0.69 & $0.62,0.77$ & 0.49 & $0.45,0.53$ \\
\hline
\end{tabular}

Ref., reference group; RRR, relative risk ratio.

areas was largest for stunting alone followed by co-morbidity, and smallest for anaemia alone. The risks of comorbidity relative to stunting alone, anaemia alone or being healthy were higher among children having a mother/main carer with lower educational level (48-80\% higher among the no education group than among those with secondary education or higher). The risks of co-morbidity relative to stunting alone, anaemia alone or being healthy were greatest among the poorest households. The magnitudes of these associations were large; relative to households in the richest quintile, the co-morbidity risks were $29-51 \%$ higher in the poorest wealth quintile. 


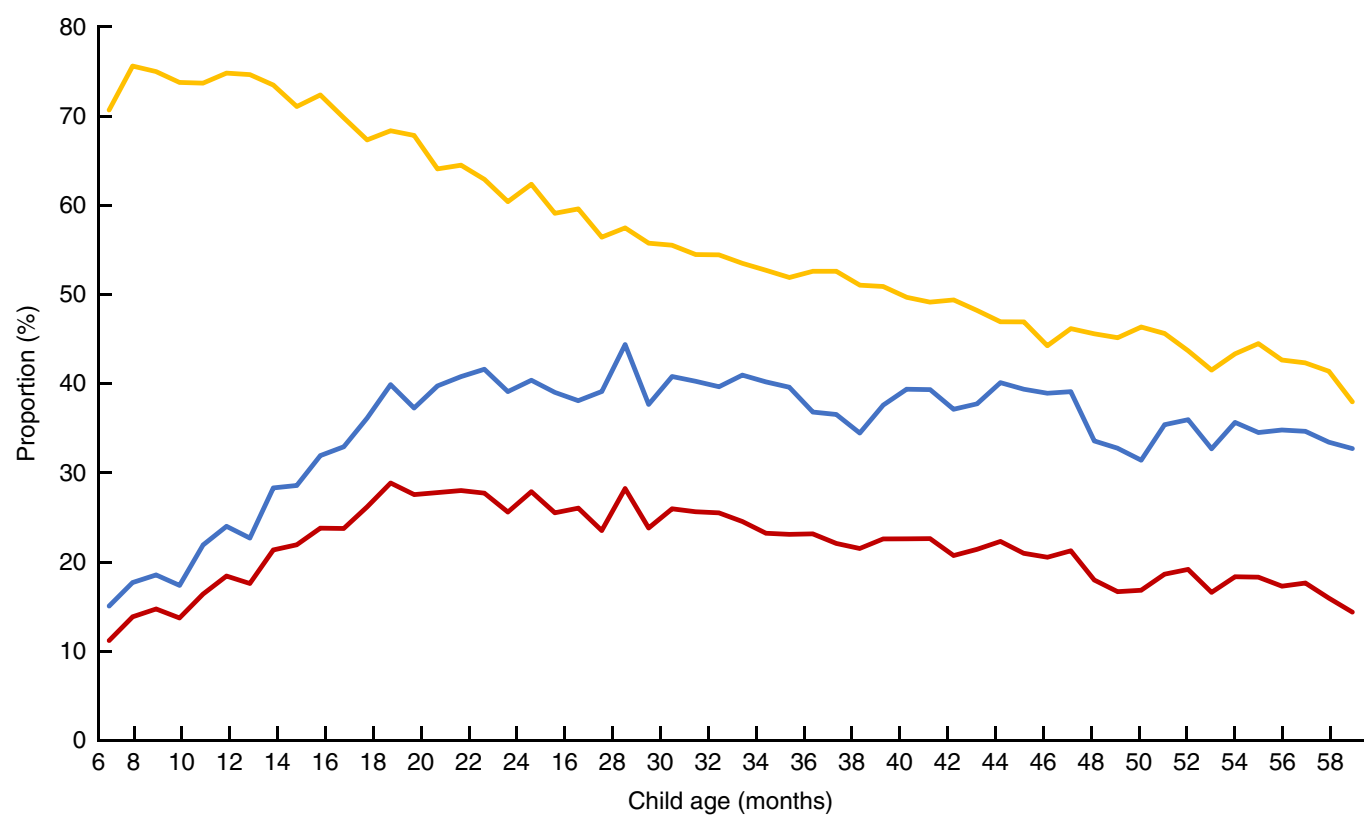

Fig. 2 (colour online) Proportions of anaemia $(-)$, stunting $(-)$ and co-morbid anaemia and stunting $(-)$, by child age, among children aged 6-59 months ( $n$ 193065) in forty-three low- and middle-income countries (pooled data from forty-three Demographic and Health Survey data sets, 2005 to 2015)

The proportions of anaemia, stunting and co-morbidity by child age are presented in Fig. 2. Overall, the proportion of anaemia was found to be at very high levels (more than $70 \%$ ) in the second half of the first year of life and to decline gradually to about $40 \%$ at 5 years of age. The proportions of stunting and co-morbidity followed a different trajectory to anaemia. The proportions were less than $15 \%$ at 6 months old, then increasing by about threefold at 18 months of age, and then decreasing consistently until 59 months of age.

\section{Inequality in child co-morbid anaemia and stunting}

Overall, a socio-economic status gradient was found in the relationship/association between anaemia, stunting, comorbidity of the two conditions and socio-economic position. Children from households in a higher socioeconomic position were less likely to have these conditions (Table 1). Among these conditions, the magnitude of these associations was highest when there was comorbidity.

The concentration indices confirmed the inequalities in those child health problems (see online supplementary material, Supplemental Table 6). The index of inequality in child co-morbid anaemia and stunting was -0.160 on average, compared with $-0 \cdot 136$ for stunting and -0.042 for anaemia.

A scatter plot (Fig. 3) of the inequality index for comorbid anaemia and stunting among children $v$. the proportion of co-morbidity by country demonstrates that the lower the proportion of co-morbidity, the higher the inequality index, except in Armenia, Kyrgyz Republic,
Egypt and Albania. Most countries had high proportions of co-morbidity (>15\%) and/or very high inequality indices $(>-0 \cdot 2)$. Some countries had very high overall proportions of the co-morbidity (>30\%) and high inequality, making the proportions among children living in the poorest quintile families more than 50\% (India and Yemen) or $40 \%$ (Mozambique, Mali, Malawi and Burkina Faso; Fig. 4). Bolivia had a high overall proportion of co-morbidity (about $20 \%$ ) and very high inequality $(-0 \cdot 3)$ resulting in the prevalence of co-morbidity among children living in the poorest quintile families to be in the group of countries with highest proportions ( $>35 \%$ ), while the proportion among children living in the richest quintile families was in the group with the lowest proportions $(<2 \%)$.

\section{Discussion}

The present study is the first to report the prevalence of co-morbid anaemia and stunting among children of preschool age in low- and middle-income countries. Overall, these conditions were found to coexist in more than one in five children in these settings. The prevalence varied among countries, ranging from rare (2.6\% in Albania) to almost one in every two children ( $43.3 \%$ in Yemen).

Data from the current study demonstrate that children living in poor countries in sub-Saharan Africa and South Asia are at highest risk for co-morbidity. These findings are consistent with previous studies. A review of 257 population-representative data sources from 107 countries worldwide from 1995 to 2011 showed that the prevalence of anaemia among children $<5$ years of age was highest in 


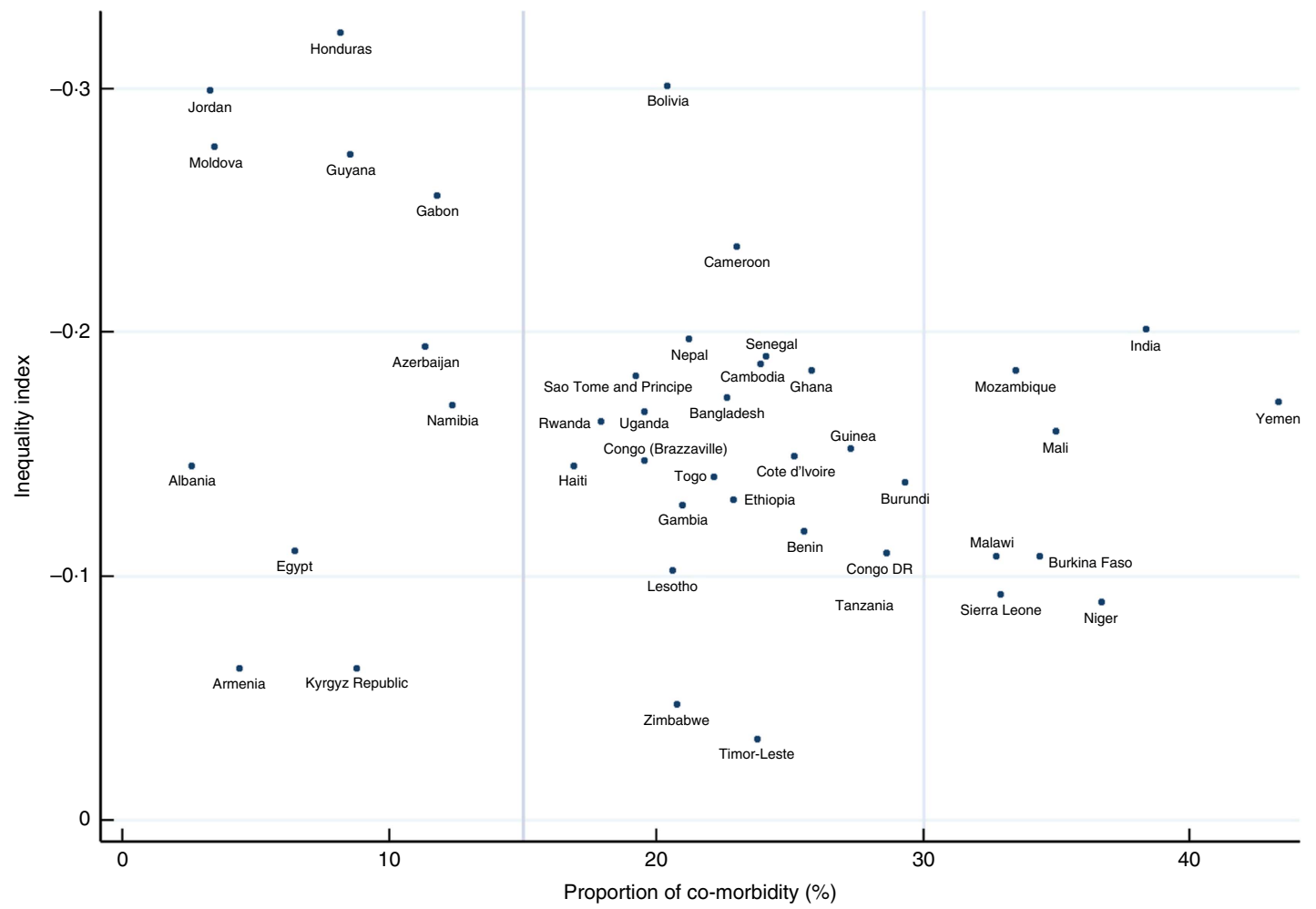

Fig. 3 (colour online) Scatter plot of the country inequality index in child co-morbid anaemia and stunting $v$. the proportion of the comorbidity among children aged 6-59 months ( $n$ 193065) in forty-three low- and middle-income countries (pooled data from fortythree Demographic and Health Survey data sets, 2005 to 2015)

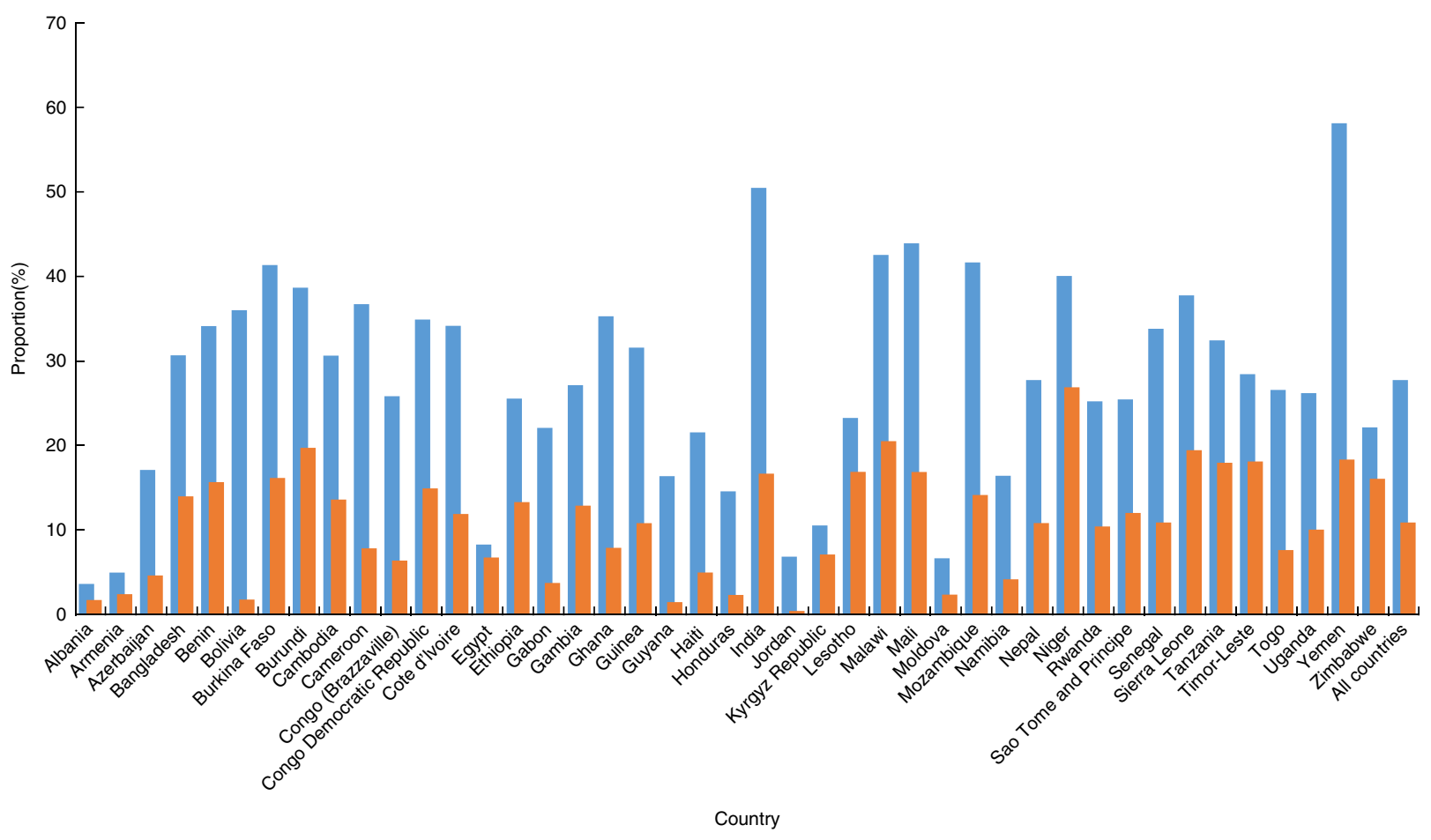

Fig. 4 (colour online) Proportions of child co-morbid anaemia and stunting in the poorest quintile ( $\square$ ) and the richest quintile groups $(\square)$, by country, among children aged 6-59 months ( $n$ 193065) in forty-three low- and middle-income countries (pooled data from forty-three Demographic and Health Survey data sets, 2005 to 2015) 
Africa and South Asia ${ }^{(15)}$. De Onis et al. ${ }^{(16)}$ reviewed 576 nationally representative surveys on child anthropometry in 148 countries from 1990 to 2010 and found that most countries with a high prevalence ( $>40 \%$ ) of stunting among children of pre-school age were located in subSaharan Africa and South Asia. Both these reviews suggest that improvements in child nutritional status in these regions over the last two decades have been modest. This may be a reflection of the economic hardship, civil wars and armed conflicts that have occurred in many countries in these regions, leading to unfavourable environments for child health and development ${ }^{(17-19)}$.

UNICEF's Causal Framework for Child Malnutrition postulates that an adverse care environment, including limited resources at both the household and community level and inadequate knowledge, influence child nutritional status via immediate causes, such as insufficient access to nutrition and exposure to infectious diseases ${ }^{(1,2)}$. Data from the current study confirm that there is a higher risk of a child having comorbid anaemia and stunting relative to having only one condition or being healthy associated with having a caregiver with limited education and living in a poor household. Overall this indicates that children living in more disadvantaged households are more likely to experience a high number of nutritional health problems than children in more advantaged households.

These data suggest that the risk of co-morbidity is higher among boys than girls. There are few available data or hypotheses that can explain this association. The constitution of breast milk varies according to whether a boy or a girl baby is being fed ${ }^{(20)}$. However, in some lowincome settings girls are breast-fed longer than boys. Obermann-Borst et al. ${ }^{(21)}$ have demonstrated that duration of breast-feeding and infant sex influence methylation of the LEPTIN gene and the subsequent growth of young children. The biopsychosocial mechanisms that underpin this lead to boys' nutritional needs being especially poorly met in the context of poverty ${ }^{(22)}$.

It was observed that the risk of co-morbidity relative to being healthy had a U-shaped association with child age such that children from 1 to 2 years of age had the highest risk. Victora et al. ${ }^{(23)}$ analysed anthropometric data of children $<5$ years of age in fifty-four countries and found that length/height-for-age of children was close to the standard in the first month of life and decreased rapidly until 24 months. Kassebaum et $a l .{ }^{(24)}$ showed that global anaemia prevalence was highest in the first year of life and reduced consistently in the rest of childhood. Those data suggest that stunting and anaemia start early and that this leads to the peak of the prevalence of co-morbidity in the second year of life. Antenatal and postnatal periods are the window of opportunity to prevent this co-morbidity occurring in childhood.

The risk of co-morbidity is different between urban and rural areas. Children living in rural have higher risk of comorbidity relative to being healthy than children living in urban areas. Living in a rural area is always a disadvantage in terms of living conditions, economic status and accessible health care compared with living in urban areas in every low- and middle-income country. These differences can influence the disparity in child health outcomes between those settings. Children living in rural areas need more support to prevent this co-morbidity.

The inequality in co-morbidity in favour of the more economically advantaged households was evident in every country in the present study but showed considerable variation that is consistent with previous studies on child malnutrition. The inequality was largest in the Latin America and Caribbean regions ${ }^{(17)}$. Greater socioeconomic inequality in a country may result in comorbidity being prevalent among poorer households even when it is uncommon among children from richer households. In many countries in the sub-Saharan African and South Asian regions, the co-morbidity is widespread among children living in poor households as the result of a large inequality combined with a high overall prevalence of co-morbidity. High socio-economic disparity in early childhood nutritional outcomes limits the ability of children to have equal opportunities to fulfil their developmental potential, leading to an ongoing, intergenerational cycle of reduced economic potential and human capital formation ${ }^{(25)}$.

The data from the present study provide health policy makers with important evidence to determine if policies need to be targeted to the most disadvantaged groups, or to be universal for the benefit of all children. These data indicate that this is not a simple decision but requires country-specific approaches. For countries with high inequality, children living in poorer households appear to require more substantial and intensive interventions especially if resources are generally limited. Alternatively, if inequality is low and the overall prevalence is high, universal interventions are indicated.

These study results suggest that co-morbid anaemia and stunting should be considered under a syndemic framework, the Childhood Anaemia and Stunting (CHAS) Syndemic. These two health problems cluster frequently among children in low- and middle-income countries and have a number of common contextual risk factors that increase the likelihood of the clustering. The syndemic framework furthers understanding of how certain individuals, families and communities are consigned to harmful environments that make them vulnerable to the syndemic. Such knowledge enables effective interventions to be implemented at policy, clinical and community levels. Comprehensive programmes to address child stunting have been developed and implemented ${ }^{(26)}$. However, interventions for the prevention and control of anaemia have focused mainly on the direct improvement of dietary intake, food fortification and supplementation with iron ${ }^{(2)}$. The syndemic approach suggests that programmes which aim to address childhood anaemia and stunting should be 
combined in resource-constrained settings and address the major risk factors for these health problems (which include food insecurity, inadequate knowledge and practices around nutrition and feeding among primary caregivers, and maternal mental health problems $)^{(6,27)}$. This is especially important for the most disadvantaged children who live in poor countries and households in which few parents are well educated. The first 1000 days of life are a crucial time for intervention when the prevalence of the co-morbidity increases rapidly and reaches its peak. The syndemic approach can cost-effectively strengthen prevention and care interventions as it considers the full scope of syndemic vulnerabilities, rather than targeting the health problems individually and ignoring the complex contexts in which they occur.

The major strength of the present study is that it includes a very large number children drawn from almost all the world's low-income countries and about a fifth from the lower-middle-income countries. These countries include those where child malnutrition is highly prevalent. The limitations of the study are that no data on anaemia were available for children under 6 months of age as they were not assessed in the DHS. Therefore, the prevalence of co-morbid anaemia and stunting among infants in this age group cannot be established. Second, data from six of the forty-three countries included in the analyses were not up to date as they had been collected prior to 2010. Finally, as it was a secondary analysis, the findings are limited by the design and the data quality of the original study.

\section{Conclusions}

The high prevalence of co-morbid child stunting and anaemia is a serious and significant public health problem in a large number of the world's low- and middle-income countries. Improved nutrition-sensitive and nutritionspecific interventions are required to address the two health problems at the same time, thereby improving the health of children and their health and productivity in adulthood, so reducing the poverty cycle in resource-poor settings.

\section{Acknowledgements}

Financial support: This work was supported by the Australian National Health and Medical Research Council (Early Career Fellowships, T.D.T. and S.Ha.); Monash University (Professorial Fellowship, J.F.); and the H and L Hecht Trust managed by Perpetual Trustees (the Jean Hailes Professorial Fellowship, J.F.). The funders of the study had no role in the study design, data collection, data analyses, data interpretation or writing of the report. The corresponding author had full access to all the data in the study and had final responsibility for the decision to submit for publication. Conflict of interest: The authors declare no conflicts of interest. Authorship: T.D.T. conceived the idea, analysed the data, and wrote and reviewed the manuscript; B.-A.B., S.Ho. and S.Ha. critically reviewed the manuscript; H.T.M.N. managed and analysed the data; J.F. wrote and reviewed the manuscript. Ethics of buman subject participation: The study was based on anonymous open data sets and was approved by the Monash University Human Research Ethics Committee (CF15/4319-2015001861).

\section{Supplementary material}

To view supplementary material for this article, please visit https://doi.org/10.1017/\$136898001800232X

\section{References}

1. Black RE, Allen LH, Bhutta ZA et al. (2008) Maternal and child undernutrition: global and regional exposures and health consequences. Lancet 371, 243-260.

2. Balarajan Y, Ramakrishnan U, Ozaltin E et al. (2011) Anaemia in low-income and middle-income countries. Lancet 378, 2123-2135.

3. Dewey KG \& Begum K (2011) Long-term consequences of stunting in early life. Matern Child Nutr 7, Suppl. 3, 5-18.

4. Singer M (2000) A dose of drugs, a touch of violence, a case of AIDS: conceptualizing the SAVA syndemic. Free Inq Creat Sociol 28, 13-24.

5. Singer M, Bulled N, Ostrach B et al. (2017) Syndemics and the biosocial conception of health. Lancet 389, 941-950.

6. Victora CG, Adair L, Fall C et al. (2008) Maternal and child undernutrition: consequences for adult health and human capital. Lancet 371, 340-357.

7. Grantham-McGregor S \& Ani C (2001) A review of studies on the effect of iron deficiency on cognitive development in children. J Nutr 131, 2S-2, 649S-666S.

8. Haas JD \& Brownlie $T$ 4th (2001) Iron deficiency and reduced work capacity: a critical review of the research to determine a causal relationship. J Nutr 131, 2S-2, 676S6885 .

9. Corsi DJ, Neuman M, Finlay JE et al. (2012) Demographic and health surveys: a profile. Int J Epidemiol 41, 1602-1613.

10. World Health Organization, UNICEF \& United Nations University (2001) Iron Deficiency Anaemia, Assessment, Prevention and Control: A Guide for Programme Managers. Geneva: WHO.

11. World Health Organization (2010) WHO Anthro for Personal Computers, Version 3.1, 2010: Software for Assessing Growth and Development of the World's Children. Geneva: WHO.

12. O'Donnell O, Doorslaer EV, Wagstaff A et al. (2008) Analyzing Health Equity Using Household Survey Data: A Guide to Techniques and Their Implementation. Washington, DC: The World Bank.

13. World Bank (2016) World Development Report 2016: Digital Dividends. Washington, DC: World Bank Publications.

14. Stata Corp (2015) Stata Survey Data Reference Manual: Release 14. College Station, TX: StataCorp LP.

15. Stevens GA, Finucane MM, De-Regil LM et al. (2013) Global, regional, and national trends in haemoglobin concentration and prevalence of total and severe anaemia in children and 
pregnant and non-pregnant women for 1995-2011: a systematic analysis of population-representative data. Lancet Glob Health 1, e16-e25.

16. De Onis M, Blossner M \& Borghi E (2012) Prevalence and trends of stunting among pre-school children, 1990-2020. Public Health Nutr 15, 142-148.

17. Van de Poel E, Hosseinpoor AR, Speybroeck N et al. (2008) Socioeconomic inequality in malnutrition in developing countries. Bull World Health Organ 86, 282-291.

18. Tran TD, Hetzel B \& Fisher J (2016) Access to iodized salt in 11 low- and lower-middle-income countries: 2000 and 2010. Bull World Health Organ 94, 122-129.

19. Tran TD, Luchters S \& Fisher J (2017) Early childhood development: impact of national human development, family poverty, parenting practices and access to early childhood education. Child Care Health Dev 43, 415-426.

20. Powe CE, Knott CD \& Conklin-Brittain N (2010) Infant sex predicts breast milk energy content. Am J Hum Biol 22, 50-54.

21. Obermann-Borst SA, Eilers PH, Tobi EW et al. (2013) Duration of breastfeeding and gender are associated with methylation of the LEPTIN gene in very young children. Pediatr Res 74, 344-349.

22. Mosha T, Laswai H \& Dakiyo S (1998) Breastfeeding, weaning practices and anthropometric status of children in Morogoro district, Tanzania. Ecol Food Nutr 37, 309-338.

23. Victora CG, de Onis M, Hallal PC et al. (2010) Worldwide timing of growth faltering: revisiting implications for interventions. Pediatrics 125, e473-e480.

24. Kassebaum NJ, Jasrasaria R, Naghavi M et al. (2014) A systematic analysis of global anemia burden from 1990 to 2010. Blood 123, 615-624.

25. Walker SP, Wachs TD, Grantham-McGregor S et al. (2011) Inequality in early childhood: risk and protective factors for early child development. Lancet $\mathbf{3 7 8}, 1325-1338$.

26. Prendergast AJ \& Humphrey JH (2014) The stunting syndrome in developing countries. Paediatr Int Child Health 34, 250-265.

27. Hanieh S, Ha TT, De Livera AM et al. (2015) Antenatal and early infant predictors of postnatal growth in rural Vietnam: a prospective cohort study. Arch Dis Child 100, 165-173. 